\title{
Development and characterization of solid lipid nanoparticles (SLNs) made of cocoa butter: a factorial design study
}

\begin{abstract}
The present study is motivated by the development of solid lipid nanoparticles (SLNs) for food applications. A focus of the study is the use of a factorial design to optimize the preparation variables. SLNs were prepared by hot homogenization at $60^{\circ} \mathrm{C}$. Cocoa butter was used to form the lipid core and the surfactant blend used to emulsify and stabilize the system was a mixture of sodium stearoyl-2-lactylate (SSL) and mono- and diglycerides of fatty acids (MDG). The particle characteristics and stability of obtained SLN-suspensions were investigated. Moreover, the effect of various cooling conditions on the properties of SLNs and the storage stability during a period of three months were examined. Results proved that cocoa butter is suitable to prepare SLNs with a food-grade quality where the optimized preparation variables resulted in a particle size of $112.7 \mathrm{~nm}$.
\end{abstract}

Keyword: High pressure homogenization; Statistical experimental design; Food nanocarriers; Sodium stearoyl-2-lactylate 